\title{
An error resilience method for depth in stereoscopic 3D video
}

\begin{abstract}
Error resilience stereoscopic 3D video can ensure robust 3D video communication especially in high error rate wireless channel. In this paper, an error resilience method is proposed for the depth data of the stereoscopic 3D video using data partitioning. Although data partitioning method is available for $2 \mathrm{D}$ video, its extension to depth information has not been investigated in the context of stereoscopic 3D video. Simulation results show that the depth data is less sensitive to error and should be partitioned towards the end of the data partitions block. The partitioned depth data is then applied to an error resilience method namely multiple description coding (MDC) to code the 2D video and the depth information. Simulation results show improved performance using the proposed depth partitioning on MDC compared to the original MDC in an error prone environment.
\end{abstract}

